Journal of Animal \& Plant Sciences, 31(5): 2021, Page: 1252-1262

ISSN (print): 1018-7081; ISSN (online): 2309-8694

https://doi.org/10.36899/JAPS.2021.5.0325

\title{
EFFECT OF DIFFERENT FAT SOURCES AND ENERGY LEVELS ON GROWTH PERFORMANCE, NUTRIENT DIGESTIBILITY AND MEAT QUALITY IN BROILER CHICKS
}

\author{
M. Shoaib*, S. A. Bhatti, H. Nawaz and M. Saif-ur-Rehman \\ Institute of Animal and Dairy Sciences, Faculty of Animal Husbandry, University of Agriculture, Faisalabad 38040, \\ Pakistan \\ *Corresponding author’s email: shoaib.imtiaz43@gmail.com
}

\begin{abstract}
This experiment was conducted to evaluate different oil sources and energy density in broiler. Two vegetable oil sources (canola and palm oil), two animal oil sources (fish oil and refined poultry oil) and two levels of energy were: recommended energy (RE); 3000 and $3200 \mathrm{kcal} / \mathrm{kg}$ according to manual of Ross 308 and $\boldsymbol{l o w}$ energy (LE); 2850 and $3050 \mathrm{kcal} / \mathrm{kg}$ used in starter and finisher phases, respectively. Four hundred and sixteen (416) day-old broiler birds were distributed in eight treatments (four replicates containing 13 birds in each) in $2 \times 4$ factorial arrangement. Feed intake, body weight and mortality were recorded. At the end of experiment, two birds from each replicate were slaughtered for carcass characteristics and meat quality parameters. Nutrient digestibility was determined using Celite ${ }^{\circledR}$ as marker. Data were subjected to statistical analysis using analysis of variance technique by completely randomized design under factorial arrangement using Minitab 17 and treatment means were compared using Tukey's Test. Results revealed that weight gain, protein efficiency ratio (PER), European production efficiency factor (EPEF), Feed efficiency (FE) and FCR were improved $(\mathrm{P} \leq 0.05)$ in birds given recommended energy diet than those fed low energy diet. Feed intake was lower $(\mathrm{P} \leq$ 0.05), whereas weight gain, PER, EPEF, FE and FCR were improved in birds given canola and poultry oil than those received fish and palm oil. Dressing percentage was higher $(\mathrm{P} \leq 0.05)$ in birds fed $\mathrm{RE}$ and poultry oil and it was lower $(\mathrm{P}$ $\leq 0.05)$ in birds fed LE and fish oil. Water holding capacity of broiler breast meat was higher $(\mathrm{P} \leq 0.05)$ in birds fed $\mathrm{RE}$ diet. Different energy levels had no effect $(\mathrm{P}>0.05)$ on dry matter $(\mathrm{DM})$, ether extract $(\mathrm{EE})$ and crude protein $(\mathrm{CP})$ digestibility (\%) at $21^{\text {st }}$ day. Birds fed diet having poultry and canola oil had greater $(\mathrm{P} \leq 0.05) \mathrm{DM}, \mathrm{CP}$ and EE digestibility on $35^{\text {th }}$ day than those fed diet having palm and fish oil. Production cost per $\mathrm{kg}$ live weight was lower $(\mathrm{P} \leq 0.05)$ in birds receiving recommended energy and poultry oil based diets. In conclusion, refined poultry oil in recommended energy had improved growth performance, meat quality and economics efficiency in broilers.
\end{abstract}

Keywords: energy density, oil sources, growth performance, meat quality, nutrient digestibility, economics efficiency

Published online first January 21, 2021 Published final August 07, 2021.

\section{INTRODUCTION}

In poultry production enterprises, feed cost accounts for around $70 \%$ of the total costs involved in production (Fan et al., 2008). Optimizing the dietary energy level, therefore, is important for lowering the feed cost per unit of poultry products (Classen, 2017). Dietary energy can be optimized for both growth performance and for enhanced meat quality. Dietary nutrient levels alter meat color, energy content, and histological makeup as well as the metabolic characteristics of broiler muscles (Zhao and Kim, 2017).

Lipids (oils and fats) are energy-rich compounds. Chemically lipids are triglycerides i.e., tri-esters of fatty acids and glycerol. Birds cannot synthesize essential fatty acids, hence; essential fatty acids are obtained from dietary fat and it must be added in poultry feed. Fat addition in broiler diet improves growth performance and also provides extra caloric effect to meet the requirements of fast-growing broilers in a short time (Baião and Lara,
2005). Digestion and absorption of nutrients are also improved by fat addition in the broiler diet. In the broiler diet, both vegetable and animal fats are used to increase the energy value (Long et al., 2018). The addition of fat in the broiler diet improves absorption of fat-soluble vitamins and enhances the likeness of bird towards feed (Liu et al., 2017).

Digestibility of fat depends on multiple properties of fat, like chain length, composition of fatty acids, ratio of saturated/unsaturated fatty acids and free fatty acids (Allahyari-Bake and Jahanian, 2017). Vegetable oils are recognized for their high content of monounsaturated fatty acids (Kiani et al., 2017). Vegetables sources are high in price and less available due to their consumption in human. Palm oil, which comes from the fruit of the Elaeis guineensis tree, is the second most common vegetable oil produced globally following soybean oil. According to Skrivan et al. (2018), palm oil is a common supplement in poultry diets. However, due to the negative impact on the environment, i.e., deforestation, 
extinction of endangered species and loss of biodiversity consumers are looking for an alternative energy source (Skrivan et al., 2018). In replacement of vegetable oil, animal oils, like poultry and fish oil, are the byproduct of rendering plants and after refining, they can be used in poultry diets as an energy source. Compared to plant source, they contain a higher degree of saturated fatty acids and omega-6 fatty acids (Liu et al., 2017). On the other hand, chicks have the ability to utilize fatty acids from unsaturated fat sources more efficiently than from saturated fat sources (Smits et al., 2000). Poultry fat contains approximately same energy than soybean oil (8220 vs $8196 \mathrm{kcal} / \mathrm{kg}$ ) (Firman et al., 2008). However, some authors stated that birds fed diet having higher saturated fatty acid had higher abdominal fat. Therefore, the present study was planned to examine the effect of different oil sources and energy levels on growth performance, nutrient digestibility and meat quality in broilers.

\section{MATERIALS AND METHODS}

The present study was conducted at Research House, Animal Nutrition Center, University of Agriculture, Faisalabad with prior approval from the animal care and use committee of the university via letter no. 15497-500.
House cleaning and preparation: Before chick arrival, the house was cleaned, washed, fumigated and closed to minimize the microbial load. Feed was offered to birds in round bottom feeder while water was available in nipple lines. House temperature was sustained at $95^{\circ} \mathrm{F}$ at first week of trial with following reduction of $5^{\circ} \mathrm{F}$ every week. Birds were vaccinated with ND+IB (day 1), IBD (day 8), IBD (day 18) and ND (day 25) vaccine. Saw dust was used as bedding material. Birds were reared maintaining all standard conditions like temperatures, relative humidity, and ventilation etc.

Experimental birds and diet: Four hundred and sixteen (416) day-old broiler birds were distributed into eight treatments with four replicates containing 13 birds in each. Eight iso-nitrogenous diets (CP 22\% in starter and 20\% in finisher phase) were formulated according to the manual of ROSS-308. Two levels of Metabolizable energy Recommended energy: $3000 \mathrm{kcal} / \mathrm{kg}$ in starter (1-21 days), $3200 \mathrm{kcal} / \mathrm{kg}$ in finisher (22-35 days) and Low energy: $2850 \mathrm{kcal} / \mathrm{kg}$ in starter, $3050 \mathrm{kcal} / \mathrm{kg}$ in finisher diet) and four sources (canola oil, palm oil, poultry oil and fish oil) were tested in a $2 \times 4$ factorial arrangement under complete randomized design. First seven days were considered as adaption period in this experiment (Table 1 and 2).

Table 1. Ingredient and nutrient composition of experimental diets during starter phase (1-21 days).

\begin{tabular}{|c|c|c|c|c|c|c|c|c|}
\hline \multirow[b]{2}{*}{ Ingredients } & \multicolumn{4}{|c|}{ LOW ENERGY } & \multicolumn{4}{|c|}{ RECOMMENDED ENERGY } \\
\hline & Canola Oil & Palm Oil & Fish Oil & Poultry Oil & Canola Oil & Palm Oil & Fish Oil & Poultry Oil \\
\hline Corn & 55.36 & 54.86 & 54.99 & 54.93 & 52.51 & 52.50 & 52.34 & 52.42 \\
\hline Soybean Meal 45\% & 37.05 & 38.42 & 38.41 & 38.42 & 38.93 & 38.93 & 38.96 & 38.95 \\
\hline Molasses & 1.70 & 0.81 & 0.70 & 0.75 & 0.00 & 0.00 & 0.00 & 0.00 \\
\hline Canola oil & 1.00 & 0.00 & 0.00 & 0.00 & 3.66 & 0.00 & 0.00 & 0.00 \\
\hline Palm Oil & 0.00 & 1.00 & 0.00 & 0.00 & 0.00 & 3.67 & 0.00 & 0.00 \\
\hline Fish Oil & 0.00 & 0.00 & 1.00 & 0.00 & 0.00 & 0.00 & 3.79 & 0.00 \\
\hline Poultry Oil & 0.00 & 0.00 & 0.00 & 1.00 & 0.00 & 0.00 & 0.00 & 3.73 \\
\hline Calcium Carbonate & 0.90 & 0.89 & 0.89 & 0.89 & 0.90 & 0.90 & 0.90 & 0.90 \\
\hline DCP & 2.15 & 2.17 & 2.17 & 2.17 & 2.17 & 2.17 & 2.17 & 2.17 \\
\hline Sodium Chloride & 0.39 & 0.37 & 0.37 & 0.37 & 0.39 & 0.39 & 0.39 & 0.39 \\
\hline Sodium & 0.30 & 0.32 & 0.31 & 0.31 & 0.30 & 0.30 & 0.30 & 0.30 \\
\hline \multicolumn{9}{|l|}{ Biocarbonate } \\
\hline L-Lysine Sulphate & 0.38 & 0.37 & 0.37 & 0.37 & 0.35 & 0.35 & 0.35 & 0.35 \\
\hline DL-Methionine & 0.36 & 0.37 & 0.37 & 0.37 & 0.37 & 0.37 & 0.37 & 0.37 \\
\hline L. Threonine & 0.11 & 0.11 & 0.11 & 0.11 & 0.11 & 0.11 & 0.11 & 0.11 \\
\hline Vitamin Premix ${ }^{*}$ & 0.15 & 0.15 & 0.15 & 0.15 & 0.15 & 0.15 & 0.15 & 0.15 \\
\hline Mineral Premix $^{* *}$ & 0.15 & 0.15 & 0.15 & 0.15 & 0.15 & 0.15 & 0.15 & 0.15 \\
\hline Total & 100 & 100 & 100 & 100 & 100 & 100 & 100 & 100 \\
\hline \multicolumn{9}{|c|}{ Nutrient composition (Calculated) } \\
\hline Dry Matter Fed & 87.35 & 87.20 & 87.22 & 87.21 & 87.61 & 87.61 & 87.62 & 87.62 \\
\hline Metab. Energy & 2850.00 & 2850.00 & 2850.00 & 2850.00 & 3000.00 & 3000.00 & 3000.00 & 3000.00 \\
\hline Crude Protein & 22.00 & 22.00 & 22.00 & 22.00 & 22.00 & 22.00 & 22.00 & 22.00 \\
\hline Ether extract & 3.32 & 3.30 & 3.30 & 3.30 & 5.87 & 5.87 & 5.98 & 5.94 \\
\hline Crude Fiber & 3.09 & 2.98 & 2.98 & 2.98 & 2.94 & 2.94 & 2.94 & 2.94 \\
\hline Ash & 4.92 & 4.96 & 4.95 & 4.96 & 4.93 & 4.91 & 4.91 & 4.93 \\
\hline Calcium & 0.96 & 0.96 & 0.96 & 0.96 & 0.96 & 0.96 & 0.96 & 0.96 \\
\hline
\end{tabular}




\begin{tabular}{lcccccccc} 
Avail. Phos. & 0.48 & 0.48 & 0.48 & 0.48 & 0.48 & 0.48 & 0.48 & 0.48 \\
Sodium & 0.25 & 0.25 & 0.25 & 0.25 & 0.25 & 0.25 & 0.25 & 0.25 \\
Potassium & 0.87 & 0.91 & 0.90 & 0.90 & 0.88 & 0.88 & 0.88 & 0.88 \\
Chlorine & 0.30 & 0.30 & 0.30 & 0.30 & 0.30 & 0.30 & 0.30 & 0.30 \\
DEB & 245.43 & 255.84 & 254.92 & 255.35 & 249.53 & 249.53 & 249.53 & 249.53 \\
Dig. Lysine & 1.28 & 1.28 & 1.28 & 1.28 & 1.28 & 1.28 & 1.28 & 1.28 \\
Dig. Methionine & 0.66 & 0.67 & 0.67 & 0.67 & 0.67 & 0.67 & 0.67 & 0.67 \\
Dig. Met + Cys & 0.95 & 0.95 & 0.95 & 0.95 & 0.95 & 0.95 & 0.95 & 0.95 \\
Dig. Threonine & 0.86 & 0.86 & 0.86 & 0.86 & 0.86 & 0.86 & 0.86 & 0.86 \\
Dig. Tryptophan & 0.25 & 0.25 & 0.25 & 0.25 & 0.25 & 0.25 & 0.25 & 0.25 \\
Dig. Arginine & 1.39 & 1.40 & 1.40 & 1.40 & 1.41 & 1.41 & 1.41 & 1.41 \\
Dig. Leucine & 1.70 & 1.70 & 1.70 & 1.70 & 1.70 & 1.70 & 1.70 & 1.70 \\
Dig. Isoleucine & 0.85 & 0.86 & 0.86 & 0.86 & 0.86 & 0.86 & 0.86 & 0.86 \\
Dig. Valine & 0.92 & 0.92 & 0.92 & 0.92 & 0.92 & 0.92 & 0.92 & 0.92 \\
Dig. Histidine & 0.53 & 0.53 & 0.53 & 0.53 & 0.53 & 0.53 & 0.53 & 0.53 \\
& & & Nutrient composition (Analyzed) & & & \\
Moisture & 89.1 & 89.0 & 88.8 & 88.5 & 88.9 & 88.1 & 88.2 & 88.0 \\
Ash & 5.4 & 5.2 & 4.8 & 4.3 & 4.4 & 4.1 & 4.6 & 4.7 \\
CP & 22.2 & 22.1 & 21.8 & 21.9 & 22.1 & 22.02 & 22.21 & 21.89 \\
EE & 3.2 & 2.9 & 3.23 & 3.12 & 5.5 & 5.3 & 5.32 & 5.1 \\
Crude fiber & 3.52 & 3.43 & 2.9 & 3.2 & 3.6 & 2.89 & 3.45 & 3.12 \\
Calcium & 0.91 & 0.9 & 0.92 & 0.93 & 0.94 & 0.91 & 0.93 & 0.92 \\
Total phosphorus & 0.58 & 0.58 & 0.55 & 0.56 & 0.58 & 0.56 & 0.57 & 0.56 \\
\hline & & & & & & &
\end{tabular}

Table 2. Ingredient and nutrient composition of experimental diets during finisher phase (22-35 days).

\begin{tabular}{|c|c|c|c|c|c|c|c|c|}
\hline \multirow[b]{2}{*}{ Ingredients } & \multicolumn{4}{|c|}{ LOW ENERGY } & \multicolumn{4}{|c|}{ RECOMMENDED ENERGY } \\
\hline & Canola Oil & Palm Oil & Fish Oil & Poultry Oil & Canola Oil & Palm Oil & Fish Oil & Poultry Oil \\
\hline Corn & 59.22 & 59.21 & 59.08 & 59.14 & 55.70 & 55.68 & 55.43 & 55.55 \\
\hline Soybean Meal $45 \%$ & 33.99 & 33.99 & 34.02 & 34.01 & 34.64 & 34.64 & 34.69 & 34.67 \\
\hline Canola oil & 3.13 & 0.00 & 0.00 & 0.00 & 6.03 & 0.00 & 0.00 & 0.00 \\
\hline Palm Oil & 0.00 & 3.14 & 0.00 & 0.00 & 0.00 & 6.04 & 0.00 & 0.00 \\
\hline Fish Oil & 0.00 & 0.00 & 3.25 & 0.00 & 0.00 & 0.00 & 6.25 & 0.00 \\
\hline Poultry Oil & 0.00 & 0.00 & 0.00 & 3.19 & 0.00 & 0.00 & 0.00 & 6.15 \\
\hline Calcium Carbonate & 0.73 & 0.73 & 0.73 & 0.73 & 0.72 & 0.72 & 0.72 & 0.72 \\
\hline DCP & 1.76 & 1.76 & 1.76 & 1.76 & 1.76 & 1.76 & 1.76 & 1.76 \\
\hline Sodium Chloride & 0.39 & 0.39 & 0.39 & 0.39 & 0.39 & 0.39 & 0.39 & 0.39 \\
\hline Sodium Biocarbonate & 0.13 & 0.13 & 0.13 & 0.13 & 0.12 & 0.12 & 0.12 & 0.12 \\
\hline L-Lysine Sulphate & 0.10 & 0.10 & 0.10 & 0.10 & 0.08 & 0.08 & 0.08 & 0.08 \\
\hline DL-Methionine & 0.26 & 0.26 & 0.26 & 0.26 & 0.26 & 0.26 & 0.26 & 0.26 \\
\hline Vitamin Premix ${ }^{*}$ & 0.15 & 0.15 & 0.15 & 0.15 & 0.15 & 0.15 & 0.15 & 0.15 \\
\hline Mineral Premix ${ }^{* *}$ & 0.15 & 0.15 & 0.15 & 0.15 & 0.15 & 0.15 & 0.15 & 0.15 \\
\hline Total & 100 & 100 & 100 & 100 & 100 & 100 & 100 & 100 \\
\hline \multicolumn{9}{|c|}{ Nutrient composition } \\
\hline Dry Matter Fed & 87.80 & 87.79 & 87.81 & 87.81 & 88.11 & 88.10 & 88.13 & 88.13 \\
\hline Metab. Energy & 3050.00 & 3050.00 & 3050.00 & 3050.00 & 3200.00 & 3200.00 & 3200.00 & 3200.00 \\
\hline Crude Protein & 20.00 & 20.00 & 20.00 & 20.00 & 20.00 & 20.00 & 20.00 & 20.00 \\
\hline Ether extract & 5.53 & 5.53 & 5.62 & 5.59 & 8.29 & 8.29 & 8.47 & 8.41 \\
\hline Crude Fiber & 2.84 & 2.84 & 2.83 & 2.84 & 2.80 & 2.80 & 2.79 & 2.80 \\
\hline Ash & 4.40 & 4.38 & 4.38 & 4.40 & 4.42 & 4.39 & 4.39 & 4.41 \\
\hline Calcium & 0.79 & 0.79 & 0.79 & 0.79 & 0.79 & 0.79 & 0.79 & 0.79 \\
\hline Avail. Phos. & 0.40 & 0.40 & 0.40 & 0.40 & 0.40 & 0.40 & 0.40 & 0.40 \\
\hline Sodium & 0.20 & 0.20 & 0.20 & 0.20 & 0.20 & 0.20 & 0.20 & 0.20 \\
\hline Potassium & 0.81 & 0.81 & 0.81 & 0.81 & 0.81 & 0.81 & 0.81 & 0.81 \\
\hline Chlorine & 0.30 & 0.30 & 0.30 & 0.30 & 0.30 & 0.30 & 0.30 & 0.30 \\
\hline DEB & 210.11 & 210.11 & 210.11 & 210.11 & 210.25 & 210.25 & 210.26 & 210.26 \\
\hline Dig. Lysine & 1.03 & 1.03 & 1.03 & 1.03 & 1.03 & 1.03 & 1.03 & 1.03 \\
\hline Dig. Methionine & 0.54 & 0.54 & 0.54 & 0.54 & 0.54 & 0.54 & 0.54 & 0.54 \\
\hline Dig. Met + Cys & 0.80 & 0.80 & 0.80 & 0.80 & 0.80 & 0.80 & 0.80 & 0.80 \\
\hline Dig. Threonine & 0.69 & 0.69 & 0.69 & 0.69 & 0.69 & 0.69 & 0.69 & 0.69 \\
\hline Dig. Tryptophan & 0.23 & 0.23 & 0.23 & 0.23 & 0.23 & 0.23 & 0.23 & 0.23 \\
\hline Dig. Arginine & 1.28 & 1.28 & 1.28 & 1.28 & 1.28 & 1.28 & 1.28 & 1.28 \\
\hline
\end{tabular}




\begin{tabular}{lcccccccc} 
Dig. Leucine & 1.60 & 1.60 & 1.60 & 1.60 & 1.59 & 1.59 & 1.59 & 1.59 \\
Dig. Isoleucine & 0.78 & 0.78 & 0.78 & 0.78 & 0.79 & 0.79 & 0.79 & 0.79 \\
Dig. Valine & 0.85 & 0.85 & 0.85 & 0.85 & 0.85 & 0.85 & 0.85 & 0.85 \\
Dig. Histidine & 0.49 & 0.49 & 0.49 & 0.49 & 0.49 & 0.49 & 0.49 & 0.49 \\
& \multicolumn{7}{c}{ Nutrient composition (Analyzed) } \\
Moisture & 88.9 & 88.92 & 90.7 & 89.7 & 89.4 & 90.23 & 88.5 & 89.1 \\
Ash & 5.52 & 5.6 & 5.3 & 5.76 & 5.23 & 5.3 & 5.7 & 5.8 \\
CP & 19.78 & 19.6 & 19.65 & 19.65 & 19.54 & 19.45 & 19.65 & 19.45 \\
EE & 4.77 & 4.87 & 5.12 & 5.23 & 7.45 & 7.32 & 7.8 & 7.92 \\
Crude fiber & 3.65 & 3.23 & 3.76 & 3.45 & 3.55 & 3.12 & 3.54 & 3.77 \\
Calcium & 0.78 & 0.76 & 0.75 & 0.78 & 0.74 & 0.76 & 0.75 & 0.77 \\
Total phosphorus & 0.54 & 0.52 & 0.52 & 0.55 & 0.51 & 0.53 & 0.54 & 0.54
\end{tabular}

${ }^{*}$ Vitamins premix provides $10000 \mathrm{IU}$ Vitamin A, $5 \mathrm{mg}$ Riboflavin, $12 \mathrm{mg}$ Ca Pantothenate, $2.2 \mathrm{mg}$ thiamin, $1.55 \mathrm{mg}$ Folic acid, $44 \mathrm{mg}$ nicotinic acid, $2.2 \mathrm{mg}$ Vitamin B6, $12.1 \mu \mathrm{g}$ Vitamin B12, $250 \mathrm{mg}$ Choline chloride, $0.11 \mathrm{mg}$ d-biotin, $1100 \mathrm{IU}$ Vitamin D3, 11.0 IU Vitamin E, $1.1 \mathrm{mg}$ Vitamin $\mathrm{K}$ per $\mathrm{kg}$ of diet.

${ }^{* *}$ Mineral premix provides $30 \mathrm{mg} \mathrm{Fe}, 50 \mathrm{mg} \mathrm{Zn,} 5 \mathrm{mg} \mathrm{Cu}, 60 \mathrm{mg} \mathrm{Mn}, 0.1 \mathrm{mg}$ Co, $0.3 \mathrm{mg} \mathrm{I}$ and $1 \mathrm{mg}$ Se per kg of diet.

\section{Data collection}

Growth performance

- $\quad$ Body weight

Body weight of birds was measured at the end of each subsequent week.

\section{- $\quad$ Feed intake}

Feed intake was calculated as follow:

Feed intake $=$ Feed offered - Feed refused

\section{- $\quad$ Feed conversion ratio}

Feed conversion ratio was calculated week-wise using the following relationship.

$$
\mathrm{FCR}=\frac{\text { Feed intake }(\mathrm{g})}{\text { Weight gain }(\mathrm{g})}
$$

\section{- $\quad$ Feed efficiency}

Feed efficiency was calculated using the

following relationship.

$$
\text { Feed eficency }=\frac{\text { Weight gain }(\mathrm{g})}{\text { Feed intake }(\mathrm{g})}
$$

\section{- $\quad$ Protein efficiency ratio}

The PER was calculated as grams of weight gain per gram of protein intake (Kamran et al., 2008).

$$
\text { Protein efficiency ratio }=\frac{\text { Weight gain }}{\text { Protein consumed }}
$$

\section{- $\quad$ European Production Efficiency Factors}

The EPEF was calculated according to the following formula (Marcu et al., 2013).

European Production Efficiency Factors

$$
=\frac{\text { Liveability } \times \text { Liveweight }(\mathrm{kg})}{\text { FCR } \times \text { Age (days) }} \times 100
$$

Nutrient digestibility: Indirect marker method was used for determining nutrient digestibility (Dourado et al., 2010). For this purpose, acid insoluble ash (Celite ${ }^{\circledR}$ ) was included in experimental diets @ 1\%. Feces were collected at $21^{\text {st }}$ and $35^{\text {th }}$ day of the experiment. Flex sheets were placed under each pen and droppings were collected three times a day. Proximate analysis of feed and feces were determined (AOAC, 2000). Nutrient digestibility was determined using following formula.
Digestibility coefficient (\%)

$$
\begin{aligned}
& =100-\left(100 \times \frac{\% \text { marker in feed }}{\% \text { marker in ileal }}\right. \\
& \left.\times \frac{\% \text { nutrient in ileal }}{\% \text { nutrient in feed }}\right)
\end{aligned}
$$

Slaughter parameters: At $35^{\text {th }}$ day, 2 birds from each replicate were slaughtered to determine the relative weights of organs including heart, gizzard and liver. Breast and thigh yield were calculated percent to carcass weight and relative organ (liver, gizzard and heart) weights and abdominal fat weight was calculated percent to live weight.

Meat quality parameters: Meat samples from breast meat were collected at the end of trial and freeze for further analysis.

pH determination: To measure $\mathrm{pH}$ of breast muscles approximately $1.5 \mathrm{~g}$ of ground breast meat was homogenized in $10 \mathrm{~mL}$ water and then measure the $\mathrm{pH}$ using pH meter (Milwaukee MW102) (Jeacocke, 1977) at $3 \mathrm{~h}$ post slaughtering.

Water holding capacity: A weighed meat sample (15gm) was centrifuged at $5000 \mathrm{rpm}$ for 15 mints at $4^{\circ} \mathrm{C}$ in a stainless tube. Water was decanted off as early to stop its re-absorption after released from meat. Meat sample was reweighed to determine liquid loss (Pearson and Dutson, 1995).

$$
\% \mathrm{WHC}=\left(\frac{\mathrm{W} 1-\mathrm{W} 2-\text { Sample weight }}{\text { Sample weight }}\right) \times 100
$$

Cooking Loss: Approximately 40 gm or 2 x $5 \mathrm{~cm}$ meat cut was taken and cooked to an internal temperature of $75 \pm$ $1^{\circ} \mathrm{C}$ in water bath $\left(80 \pm 0.5^{\circ} \mathrm{C}\right)$ for 30 to 35 minutes. After cooling the meat, cooking loss was calculated as weight loss from meat (Ahmed et al., 2015).

Quality test of oil: Quality of oil was measure in term of moisture, percentage of free fatty acids and peroxide values (Butolo, 2002) (table 3). 
Table 3. Lab analysis of oil used in experimental diets.

\begin{tabular}{lccc}
\hline & $\begin{array}{c}\text { Moisture } \\
\mathbf{( \% )}\end{array}$ & $\begin{array}{c}\text { Free fatty } \\
\text { acid (\%) }\end{array}$ & $\begin{array}{c}\text { Per oxidize } \\
\text { value (\%) }\end{array}$ \\
\hline Canola oil & 0.02 & 0.94 & 1.81 \\
Palm oil & 0.06 & 0.84 & 2.09 \\
Refined poultry & 1.08 & 0.82 & 2.91 \\
oil & & & \\
Fish oil & 0.08 & 25.2 & 21.9 \\
\hline
\end{tabular}

Statistical Analysis: The obtained data was subjected to statistical analysis using analysis of variance technique under completely randomized design factorial arrangement using Minitab 17 and treatment means were compared using Tukey's Test (Steel et al., 1997).

\section{RESULTS}

Effect of energy levels and oil sources on growth performance in broiler

Starter phase: Weight gain, feed intake (FI), FCR, PER, $\mathrm{FE}$ and EPEF of starter phase are given in table 4. Feed intake, WG, FCR, PER, FCR, FE and EPEF were not influenced $(P>0.05)$ by different oil sources and energy levels. There was no interaction $(\mathrm{P}>0.05)$ between energy levels and oil sources on growth performance and nutrient utilization in starter period.
Finisher phase: Fed intake, WG, FCR, PER and EPEF of finisher phase are given in table 5. Weight gain, PER, FE, EPEF and FCR were improved $(\mathrm{P} \leq 0.05)$ in birds fed RE diet than those fed LE diet. Feed intake was lower $(\mathrm{P} \leq$ 0.05), whereas, PER, EPEF, FE and FCR were improved $(\mathrm{P} \leq 0.05)$ in birds received diet having canola oil and refined poultry oil than those fed palm and fish oil. However, WG was not influenced $(\mathrm{P}>0.05)$ different oil sources. Improved $(\mathrm{P} \leq 0.05) \mathrm{FCR}, \mathrm{PER}, \mathrm{FE}$ and EPEF were recorded in birds received diet having poultry oil and recommended energy diet and poor FCR, PER and EPEF was observed in birds received diet having low energy and palm oil.

Whole life: The results of total FI, WG, FCR PER, FE and EPEF are given in table 6. Weight gain, PER, EPEF, FE and FCR were improved $(\mathrm{P} \leq 0.05)$ in birds received $\mathrm{RE}$ diet than those fed LE diet. Feed intake was lower $(\mathrm{P} \leq$ 0.05), WG, PER, EPEF, FE and FCR were improved ( $\mathrm{P} \leq$ 0.05 ) in birds fed canola and poultry oil based diet than those received diet having fish and palm oil. There was an interaction $(\mathrm{P} \leq 0.05)$ between energy level and oil sources on growth performance. Birds fed diet having refined poultry oil in RE diet had higher $(\mathrm{P} \leq 0.05)$ weight gain, PER, FE, EPEF and better $(\mathrm{P} \leq 0.05) \mathrm{FCR}$, while, higher $(\mathrm{P} \leq 0.05)$ feed intake, lower $(\mathrm{P} \leq 0.05) \mathrm{PER}$ and poor $(\mathrm{P}$ $\leq 0.05)$ FCR was observed in birds having palm oil in low energy diet.

Table 4. Effect of energy levels and oil sources on growth performance during starter phase (8-21 days).

\begin{tabular}{|c|c|c|c|c|c|c|}
\hline & Feed Intake (g) & Weight gain (g) & FCR (FI:WG) & $\begin{array}{l}\text { Feed } \\
\text { efficiency } \\
\text { (WG:FI) }\end{array}$ & PER & EPEF \\
\hline \multicolumn{7}{|l|}{ Energy Density } \\
\hline $\mathrm{RE}$ & 1112.25 & 601.27 & 1.866 & 0.54 & 2.46 & 286.15 \\
\hline LE & 1135.18 & 611.78 & 1.864 & 0.54 & 2.46 & 286.30 \\
\hline SEM & 20.5 & 13.3 & 0.06 & 0.02 & 0.07 & 12.7 \\
\hline$P$ Value & 0.438 & 0.583 & 0.976 & 0.902 & 0.945 & 0.993 \\
\hline \multicolumn{7}{|l|}{ Oil Sources } \\
\hline Canola Oil & 1123.72 & 611.72 & 1.852 & 0.55 & 2.46 & 287.82 \\
\hline Fish Oil & 1117.72 & 602.69 & 1.853 & 0.54 & 2.45 & 285.55 \\
\hline Palm Oil & 1147.62 & 592.70 & 1.942 & 0.52 & 2.35 & 269.58 \\
\hline Poultry Oil & 1105.80 & 618.99 & 1.812 & 0.57 & 2.58 & 301.96 \\
\hline SEM & 29.0 & 18.9 & 0.08 & 0.02 & 0.09 & 18.0 \\
\hline$P$ Value & 0.777 & 0.780 & 0.687 & 0.463 & 0.420 & 0.657 \\
\hline \multicolumn{7}{|c|}{ Energy Density x Oil Sources } \\
\hline RE Canola Oil & 1117.92 & 602.37 & 1.869 & 0.54 & 2.44 & 280.70 \\
\hline RE Fish Oil & 1124.36 & 606.35 & 1.851 & 0.54 & 2.46 & 284.15 \\
\hline RE Palm Oil & 1120.95 & 573.23 & 1.966 & 0.51 & 2.30 & 263.57 \\
\hline RE Poultry Oil & 1085.78 & 623.13 & 1.778 & 0.58 & 2.65 & 316.17 \\
\hline LE Canola Oil & 1129.52 & 621.06 & 1.834 & 0.55 & 2.49 & 294.94 \\
\hline LE Fish Oil & 1111.08 & 599.03 & 1.855 & 0.54 & 2.44 & 286.94 \\
\hline LE Palm Oil & 1174.29 & 612.18 & 1.918 & 0.52 & 2.39 & 275.59 \\
\hline LE Poultry Oil & 1125.81 & 614.85 & 1.847 & 0.55 & 2.51 & 287.74 \\
\hline SEM & 41.1 & 26.7 & 0.11 & 0.03 & 0.14 & 25.4 \\
\hline$P$ Value & 0.852 & 0.781 & 0.952 & 0.889 & 0.853 & 0.825 \\
\hline
\end{tabular}

SEM, standard error of the mean;

$\mathrm{P}>0.05$ : Non-Significant, $\mathrm{P} \leq 0.05$ : Significant 
Table 5. Effect of energy levels and oil sources on growth performance during finisher phase (22-35 days).

\begin{tabular}{|c|c|c|c|c|c|c|}
\hline & Feed Intake (g) & Weight gain (g) & FCR (FI:WG) & $\begin{array}{c}\text { Feed } \\
\text { efficiency } \\
\text { (WG:FI) }\end{array}$ & PER & EPEF \\
\hline \multicolumn{7}{|l|}{ Energy Density } \\
\hline $\mathrm{RE}$ & 2067.91 & $1219.23^{\mathrm{a}}$ & $1.704^{\mathrm{b}}$ & $0.59^{\mathrm{a}}$ & $3.03^{\mathrm{a}}$ & $494.88^{\mathrm{a}}$ \\
\hline LE & 2094.85 & $1156.26^{\mathrm{b}}$ & $1.811^{\mathrm{a}}$ & $0.56^{b}$ & $2.82^{\mathrm{b}}$ & $458.37^{b}$ \\
\hline SEM & 28.9 & 16.4 & 0.02 & 0.01 & 0.04 & 12.5 \\
\hline$P$ Value & 0.516 & 0.012 & 0.003 & 0.003 & 0.001 & 0.050 \\
\hline \multicolumn{7}{|l|}{ Oil Sources } \\
\hline Canola Oil & $1978.78^{b}$ & 1202.11 & $1.647^{\mathrm{b}}$ & $0.61^{\mathrm{a}}$ & $3.09^{\mathrm{a}}$ & $522.48^{\mathrm{a}}$ \\
\hline Fish Oil & $2187.21^{\mathrm{a}}$ & 1186.48 & $1.845^{\mathrm{a}}$ & $0.54^{b}$ & $2.78^{\mathrm{b}}$ & $435.59^{b}$ \\
\hline Palm Oil & $2131.27^{\mathrm{ab}}$ & 1148.84 & $1.855^{\mathrm{a}}$ & $0.54^{\mathrm{b}}$ & $2.75^{\mathrm{b}}$ & $437.99^{b}$ \\
\hline Poultry Oil & $2028.27^{\mathrm{ab}}$ & 1213.54 & $1.683^{\mathrm{b}}$ & $0.60^{\mathrm{a}}$ & $3.07^{\mathrm{a}}$ & $510.43^{\mathrm{a}}$ \\
\hline SEM & 40.9 & 23.2 & 0.03 & 0.01 & 0.06 & 17.7 \\
\hline P Value & 0.006 & 0.246 & 0.0001 & 0.0001 & 0.0001 & 0.002 \\
\hline \multicolumn{7}{|c|}{ Energy Density x Oil Sources } \\
\hline RE Canola Oil & $2095.95^{\mathrm{abc}}$ & 1264.15 & $1.661^{\mathrm{bcd}}$ & $0.60^{\mathrm{ab}}$ & $3.09^{\mathrm{ab}}$ & $545.31^{\mathrm{ab}}$ \\
\hline RE Fish Oil & $2185.74^{\mathrm{ab}}$ & 1185.86 & $1.846^{\mathrm{ab}}$ & $0.54^{\mathrm{bc}}$ & $2.79^{\mathrm{bc}}$ & $410.73^{c}$ \\
\hline RE Palm Oil & $2030.49^{\mathrm{abc}}$ & 1147.37 & $1.771^{\mathrm{abc}}$ & $0.57^{\mathrm{bc}}$ & $2.88^{\mathrm{bc}}$ & $452.29^{\mathrm{bc}}$ \\
\hline RE Poultry Oil & $1959.47^{\mathrm{bc}}$ & 1279.52 & $1.537^{\mathrm{d}}$ & $0.65^{\mathrm{a}}$ & $3.36^{\mathrm{a}}$ & $571.18^{\mathrm{a}}$ \\
\hline LE Canola Oil & $1861.60^{\mathrm{c}}$ & 1140.07 & $1.633^{\mathrm{cd}}$ & $0.61^{\mathrm{ab}}$ & $3.10^{\mathrm{ab}}$ & $499.66^{\mathrm{bc}}$ \\
\hline LE Fish Oil & $2188.68^{\mathrm{ab}}$ & 1187.10 & $1.843^{\mathrm{abc}}$ & $0.54^{\mathrm{bc}}$ & $2.77^{\mathrm{bc}}$ & $460.45^{\mathrm{abc}}$ \\
\hline LE Palm Oil & $2232.04^{\mathrm{a}}$ & 1150.31 & $1.939^{\mathrm{a}}$ & $0.52^{\mathrm{c}}$ & $2.63^{\mathrm{c}}$ & $423.68^{c}$ \\
\hline LE Poultry Oil & $2097.08^{\mathrm{abc}}$ & 1147.56 & $1.829^{\mathrm{abc}}$ & $0.55^{\mathrm{bc}}$ & $2.79^{\mathrm{bc}}$ & $449.68^{\mathrm{abc}}$ \\
\hline$S E M$ & 57.8 & 32.8 & 0.05 & 0.01 & 0.08 & 25.0 \\
\hline$P$ Value & 0.005 & 0.074 & 0.005 & 0.004 & 0.004 & 0.02 \\
\hline
\end{tabular}

SEM, standard error of the mean;

$\mathrm{P}>0.05$ : Non-Significant, $\mathrm{P} \leq 0.05$ : Significant

Table 6. Effect of energy levels and oil sources on growth performance (8-35 days).

\begin{tabular}{|c|c|c|c|c|c|c|}
\hline & Feed Intake (g) & Weight gain (g) & FCR (FI:WG) & $\begin{array}{c}\text { Feed } \\
\text { efficiency } \\
\text { (WG:FI) }\end{array}$ & PER & EPEF \\
\hline \multicolumn{7}{|l|}{ Energy Density } \\
\hline $\mathrm{RE}$ & 3187.10 & $1820.49^{a}$ & $1.755^{\mathrm{b}}$ & $0.57^{\mathrm{a}}$ & $2.81^{\mathrm{a}}$ & $389.80^{\mathrm{a}}$ \\
\hline LE & 3230.02 & $1768.04^{\mathrm{b}}$ & $1.827^{\mathrm{a}}$ & $0.55^{\mathrm{b}}$ & $2.68^{\mathrm{b}}$ & $369.60^{\mathrm{b}}$ \\
\hline$S E M$ & 30.3 & 12.6 & 0.02 & 0.001 & 0.03 & 6.82 \\
\hline$P$ Value & 0.326 & 0.007 & 0.013 & 0.015 & 0.005 & 0.047 \\
\hline \multicolumn{7}{|l|}{ Oil Sources } \\
\hline Canola Oil & $3116.37^{\mathrm{b}}$ & $1813.83^{\mathrm{a}}$ & $1.719^{\mathrm{b}}$ & $0.58^{\mathrm{a}}$ & $2.85^{\mathrm{a}}$ & $414.49^{\mathrm{a}}$ \\
\hline Fish Oil & $3304.92^{\mathrm{a}}$ & $1789.17^{\mathrm{ab}}$ & $1.848^{\mathrm{a}}$ & $0.54^{\mathrm{b}}$ & $2.66^{\mathrm{b}}$ & $351.11^{\mathrm{b}}$ \\
\hline Palm Oil & $3278.88^{\mathrm{ab}}$ & $1741.54^{\mathrm{b}}$ & $1.883^{\mathrm{a}}$ & $0.53^{\mathrm{b}}$ & $2.60^{\mathrm{b}}$ & $355.28^{\mathrm{b}}$ \\
\hline Poultry Oil & $3134.07^{\mathrm{b}}$ & $1832.53^{\mathrm{a}}$ & $1.715^{\mathrm{b}}$ & $0.59^{\mathrm{a}}$ & $2.88^{\mathrm{a}}$ & $397.91^{\mathrm{a}}$ \\
\hline$S E M$ & 42.8 & 17.8 & 0.03 & 0.001 & 0.04 & 9.65 \\
\hline$P$ Value & 0.007 & 0.008 & 0.0001 & 0.0001 & 0.0001 & 0.0001 \\
\hline \multicolumn{7}{|c|}{ Energy Density x Oil Sources } \\
\hline RE Canola Oil & $3241.62^{\mathrm{abc}}$ & $1866.52^{\mathrm{ab}}$ & $1.738^{\mathrm{bc}}$ & $0.58^{\mathrm{abc}}$ & $2.84^{\mathrm{ab}}$ & $422.60^{\mathrm{ab}}$ \\
\hline RE Fish Oil & $3310.10^{\mathrm{ab}}$ & $1792.21^{\mathrm{abc}}$ & $1.848^{\mathrm{ab}}$ & $0.54^{\mathrm{bc}}$ & $2.67^{\mathrm{bc}}$ & $327.51^{\mathrm{d}}$ \\
\hline RE Palm Oil & $3151.44^{\mathrm{abc}}$ & $1720.59^{c}$ & $1.835^{\mathrm{ab}}$ & $0.55^{\mathrm{bc}}$ & $2.66^{\mathrm{bc}}$ & $367.67^{\mathrm{bcd}}$ \\
\hline RE Poultry Oil & $3045.25^{\mathrm{bc}}$ & $1902.65^{\mathrm{a}}$ & $1.601^{\mathrm{c}}$ & $0.63^{\mathrm{a}}$ & $3.08^{\mathrm{a}}$ & $441.40^{\mathrm{a}}$ \\
\hline LE Canola Oil & $2991.12^{\mathrm{c}}$ & $1761.13^{\mathrm{bc}}$ & $1.700^{\mathrm{bc}}$ & $0.59^{\mathrm{ab}}$ & $2.85^{\mathrm{ab}}$ & $406.38^{\mathrm{abc}}$ \\
\hline LE Fish Oil & $3299.75^{\mathrm{ab}}$ & $1786.13^{\mathrm{abc}}$ & $1.847^{\mathrm{ab}}$ & $0.54^{\mathrm{bc}}$ & $2.65^{\mathrm{bc}}$ & $374.72^{\text {bcd }}$ \\
\hline LE Palm Oil & $3406.33^{\mathrm{a}}$ & $1762.49^{\mathrm{bc}}$ & $1.932^{\mathrm{a}}$ & $0.52^{\mathrm{c}}$ & $2.54^{\mathrm{c}}$ & $342.88^{\mathrm{cd}}$ \\
\hline LE Poultry Oil & $3222.89^{a b c}$ & $1762.41^{\mathrm{bc}}$ & $1.829^{\mathrm{ab}}$ & $0.55^{\mathrm{bc}}$ & $2.68^{\mathrm{bc}}$ & $354.42^{\mathrm{cd}}$ \\
\hline SEM & 60.5 & 25.1 & 0.04 & 0.01 & 0.06 & 13.6 \\
\hline$P$ Value & 0.002 & 0.004 & 0.008 & 0.005 & 0.01 & 0.001 \\
\hline
\end{tabular}

SEM, standard error of the mean;

$\mathrm{P}>0.05$ : Non-Significant, $\mathrm{P} \leq 0.05$ : Significant 
Effect of energy levels and oil sources on carcass characteristics in broiler: The results of carcass characteristics are given in table 7 . Dressing percentage was higher $(\mathrm{P} \leq 0.05)$ in birds fed $\mathrm{RE}$ diet than those fed LE diet. However, dressing percentage was not affected ( $P$ $>0.05)$ by different oil sources. There was no interaction $(\mathrm{P}>0.05)$ between energy levels and oil sources on dressing percentage. Breast yield (including wings and neck), thigh yield (included drumstick), heart weight and gizzard weight were not different in birds fed different energy levels and oil sources. Birds received diet containing fish oil had higher $(\mathrm{P} \leq 0.05)$ liver weight and abdominal fat deposition than those fed diet having canola, palm and poultry oil. Abdominal fat deposition and liver weight were higher $(\mathrm{P} \leq 0.05)$ in birds received diet having low energy and fish oil.

Table 7. Effect of energy levels and oil sources on carcass characteristics.

\begin{tabular}{|c|c|c|c|c|c|c|c|}
\hline & $\begin{array}{c}\text { Dressing } \\
\text { percentage }(\%)\end{array}$ & $\begin{array}{c}\text { Breast } \\
\text { yield } \\
(\%)^{*}\end{array}$ & $\begin{array}{l}\text { Thigh } \\
\text { yield } \\
(\%) *\end{array}$ & $\begin{array}{c}\text { Heart weight } \\
(\%)^{* * *}\end{array}$ & $\begin{array}{c}\text { Gizzard } \\
\text { weight }(\%)^{* *}\end{array}$ & $\begin{array}{l}\text { Liver weight } \\
(\%)^{* *}\end{array}$ & $\begin{array}{l}\text { Abdominal } \\
\text { fat }(\%)^{* *}\end{array}$ \\
\hline \multicolumn{8}{|l|}{ Energy Density } \\
\hline $\mathrm{RE}$ & $63.34^{\mathrm{a}}$ & 61.83 & 38.17 & 0.66 & 1.45 & 2.51 & 1.53 \\
\hline $\mathrm{LE}$ & $61.44^{\mathrm{b}}$ & 61.43 & 38.57 & 0.60 & 1.49 & 2.64 & 1.50 \\
\hline SEM & 0.51 & 0.25 & 0.25 & 0.03 & 0.06 & 0.07 & 0.06 \\
\hline P Value & 0.011 & 0.26 & 0.26 & 0.16 & 0.56 & 0.16 & 0.711 \\
\hline \multicolumn{8}{|l|}{ Oil Sources } \\
\hline Canola Oil & 62.75 & 61.58 & 38.42 & 0.64 & 1.49 & $2.54^{\mathrm{ab}}$ & $1.50^{\mathrm{ab}}$ \\
\hline Palm Oil & 61.83 & 61.59 & 38.41 & 0.58 & 1.42 & $2.32^{b}$ & $1.35^{\mathrm{b}}$ \\
\hline Fish Oil & 61.59 & 61.67 & 38.33 & 0.60 & 1.45 & $2.84^{\mathrm{a}}$ & $1.68^{\mathrm{a}}$ \\
\hline Poultry Oil & 63.38 & 61.69 & 38.31 & 0.69 & 1.52 & $2.61^{\mathrm{ab}}$ & $1.54^{\mathrm{ab}}$ \\
\hline SEM & 0.73 & 0.35 & 0.35 & 0.04 & 0.08 & 0.09 & 0.08 \\
\hline P Value & 0.28 & 0.99 & 0.99 & 0.19 & 0.79 & 0.003 & 0.045 \\
\hline \multicolumn{8}{|c|}{ Energy Density x Oil Sources } \\
\hline RE Canola Oil & 62.91 & 61.61 & 38.39 & 0.64 & 1.43 & $2.66^{\mathrm{ab}}$ & $1.51^{\mathrm{ab}}$ \\
\hline RE Palm Oil & 62.68 & 62.00 & 38.00 & 0.60 & 1.47 & $2.30^{\mathrm{b}}$ & $1.46^{\mathrm{ab}}$ \\
\hline RE Fish Oil & 63.56 & 61.86 & 38.14 & 0.65 & 1.45 & $2.53^{\mathrm{b}}$ & $1.59^{\mathrm{ab}}$ \\
\hline RE Poultry Oil & 64.19 & 61.86 & 38.14 & 0.73 & 1.45 & $2.55^{\mathrm{b}}$ & $1.57^{\mathrm{ab}}$ \\
\hline LE Canola Oil & 62.58 & 61.54 & 38.46 & 0.63 & 1.56 & $2.42^{\mathrm{b}}$ & $1.49^{\mathrm{ab}}$ \\
\hline LE Palm Oil & 60.99 & 61.18 & 38.82 & 0.56 & 1.38 & $2.34^{\mathrm{b}}$ & $1.25^{\mathrm{b}}$ \\
\hline LE Fish Oil & 59.62 & 61.47 & 38.53 & 0.55 & 1.44 & $3.15^{\mathrm{a}}$ & $1.77^{\mathrm{a}}$ \\
\hline LE Poultry Oil & 62.57 & 61.53 & 38.47 & 0.66 & 1.60 & $2.67^{\mathrm{ab}}$ & $1.50^{\mathrm{ab}}$ \\
\hline SEM & 1.03 & 0.50 & 0.50 & 0.06 & 0.11 & 0.13 & 0.11 \\
\hline P Value & 0.37 & 0.90 & 0.90 & 0.85 & 0.67 & 0.018 & 0.38 \\
\hline
\end{tabular}

SEM, standard error of the mean;

$\mathrm{P}>0.05$ : Non-Significant, $\mathrm{P} \leq 0.05$ : Significant

${ }^{*}$ Breast and thigh yield ( $\%$ to carcass weight)

** Relative organ (liver, gizzard and heart) weight and abdominal fat ( $\%$ to live weight)

Effect of energy levels and oil sources on meat quality parameters in broiler: Results of breast meat quality parameters are given in table 8 . Water holding capacity or broiler breast meat was higher $(\mathrm{P} \leq 0.05)$ in birds fed diets with RE compared to those fed LE diet. Higher $(\mathrm{P} \leq 0.05)$ water holding capacity was observed in birds fed diet containing canola oil. There was an interaction $(\mathrm{P} \leq 0.05)$ between energy levels and oil sources on water holding capacity. Cooking losses and $\mathrm{pH}$ of the breast meat were not affected $(\mathrm{P}>0.05)$ by energy levels and different oil sources. There was no interaction $(\mathrm{P}>0.05)$ between energy levels and different oil sources in cooking loss and $\mathrm{pH}$.

Effect of energy levels and oil sources on nutrient digestibility in broiler at 21 day: Results of nutrient digestibility at $21^{\text {st }}$ day are given in table 9 . Birds fed diet having recommended and lower energy had no effect $(\mathrm{P}>$
0.05) on DM, EE and CP digestibility (\%). Further, different oil sources (canola, palm, poultry and fish oil) had no effect $(\mathrm{P}>0.05)$ on DM, EE and CP digestibility $(\%)$ at $21^{\text {st }}$ day. However, DM digestibility (\%) was higher $(\mathrm{P} \leq 0.05)$ in birds fed poultry oil and recommended energy while it was lower $(\mathrm{P} \leq 0.05)$ in birds received diet having poultry oil and low energy.

Effect of energy levels and oil sources on nutrient digestibility in broiler at 35 day: Results of nutrient digestibility at $35^{\text {th }}$ day are given in table 9 . Birds fed diet having recommended and lower energy had no effect $(\mathrm{P}>$ 0.05 ) on DM, EE and CP digestibility (\%). However, birds fed diet having poultry and canola oil had greater $(\mathrm{P} \leq$ $0.05) \mathrm{DM}, \mathrm{CP}$ and EE digestibility (\%) at $35^{\text {th }}$ day than those fed diet having palm and fish oil. Highest $(\mathrm{P} \leq 0.05)$ $\mathrm{DM}$ and EE digestibility were observed in birds received diet having poultry oil with recommended energy. Highest 
$(\mathrm{P} \leq 0.05) \mathrm{CP}$ digestibility was observed in birds offered diet having canola oil with recommended energy and lowest $(\mathrm{P} \leq 0.05) \mathrm{CP}$ digestibility was shown in birds offered diet having palm oil with low energy.
Effect of energy levels and oil sources on economics performance in broiler: Poultry oil-based diet had lower $(\mathrm{P} \leq 0.05)$ production cost per $\mathrm{kg}$ than others oils (Table 10). Further, birds fed diet having recommended energy and poultry oil had lower $(\mathrm{P} \leq 0.05)$ production cost per $\mathrm{kg}$ than fish oil and recommended energy (Table 11).

Table 8. Effect of energy levels and oil sources on meat quality parameters.

\begin{tabular}{|c|c|c|c|}
\hline & WHC (\%) & Cooking loss (\%) & pH \\
\hline \multicolumn{4}{|l|}{ Energy Density } \\
\hline RE & $60.38^{\mathrm{a}}$ & 25.27 & 6.05 \\
\hline LE & $55.45^{\mathrm{b}}$ & 27.33 & 6.03 \\
\hline SEM & 1.12 & 0.89 & 0.03 \\
\hline$P$ Value & 0.005 & 0.124 & 0.681 \\
\hline \multicolumn{4}{|l|}{ Oil Sources } \\
\hline Canola Oil & $63.31^{\mathrm{a}}$ & 25.88 & 6.11 \\
\hline Fish Oil & $53.31^{\mathrm{c}}$ & 27.60 & 6.09 \\
\hline Palm Oil & $54.93^{\mathrm{bc}}$ & 24.60 & 5.96 \\
\hline Poultry Oil & $60.11^{\mathrm{ab}}$ & 27.13 & 6.00 \\
\hline SEM & 1.59 & 1.27 & 0.05 \\
\hline P Value & 0.001 & 0.364 & 0.120 \\
\hline \multicolumn{4}{|c|}{ Energy Density x Oil Sources } \\
\hline RE Canola Oil & $65.89^{\mathrm{a}}$ & 24.97 & 6.17 \\
\hline RE Fish Oil & $56.13^{\mathrm{abc}}$ & 25.72 & 6.04 \\
\hline RE Palm Oil & $57.04^{\mathrm{abc}}$ & 23.63 & 6.02 \\
\hline RE Poultry Oil & $62.46^{\mathrm{ab}}$ & 26.76 & 5.97 \\
\hline LE Canola Oil & $60.73^{a b c}$ & 26.78 & 6.05 \\
\hline LE Fish Oil & $50.49^{\mathrm{abc}}$ & 29.48 & 6.14 \\
\hline LE Palm Oil & $52.82^{\mathrm{bc}}$ & 25.57 & 5.91 \\
\hline LE Poultry Oil & $57.75^{\mathrm{c}}$ & 27.50 & 6.03 \\
\hline SEM & 2.25 & 1.80 & 0.07 \\
\hline P Value & 0.990 & 0.864 & 0.278 \\
\hline
\end{tabular}

SEM, standard error of the mean; WHC: water holding capacity

$\mathrm{P}>0.05$ : Non-Significant, $\mathrm{P} \leq 0.05$ : Significant

Table 9. Effect of energy levels and oil sources on nutrient digestibility in broiler.

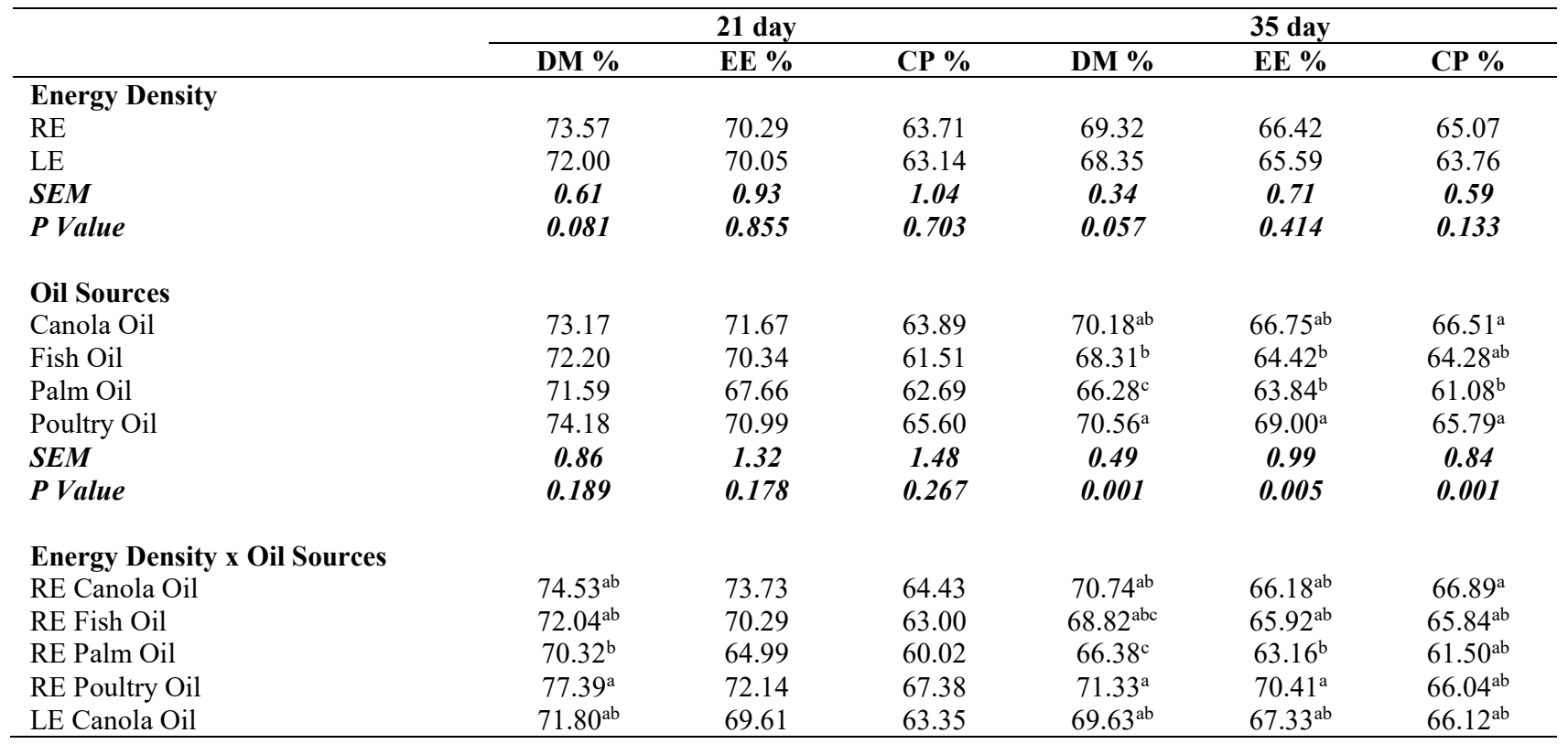




\begin{tabular}{|c|c|c|c|c|c|c|}
\hline LE Fish Oil & $72.36^{\mathrm{ab}}$ & 70.39 & 60.02 & $67.80^{\mathrm{bc}}$ & $62.92^{\mathrm{b}}$ & $62.73^{\mathrm{ab}}$ \\
\hline LE Palm Oil & $72.86^{\mathrm{ab}}$ & 70.33 & 65.36 & $66.19^{c}$ & $64.51^{\mathrm{ab}}$ & $60.65^{\mathrm{b}}$ \\
\hline LE Poultry Oil & $70.96^{b}$ & 69.85 & 63.82 & $69.78^{\mathrm{ab}}$ & $67.59^{\mathrm{ab}}$ & $65.55^{\mathrm{ab}}$ \\
\hline SEM & 1.22 & 1.86 & 2.09 & 0.69 & 1.41 & 1.19 \\
\hline P Value & 0.007 & 0.09 & 0.155 & 0.795 & 0.253 & 0.668 \\
\hline
\end{tabular}

SEM, standard error of the mean;

$\mathrm{P}>0.05$ : Non-Significant, $\mathrm{P} \leq 0.05$ : Significant

Table 10. Effect of energy levels and oil sources on economics efficiency (Main effect).

\begin{tabular}{|c|c|c|c|c|c|c|c|c|c|c|}
\hline \multirow{2}{*}{$\begin{array}{l}\text { Production } \\
\text { Cost (Rs.) }\end{array}$} & \multicolumn{2}{|c|}{ Energy levels } & \multirow[b]{2}{*}{ SEM } & \multirow[b]{2}{*}{$\begin{array}{c}\text { P- } \\
\text { value }\end{array}$} & \multicolumn{4}{|c|}{ Oil Sources } & \multirow[b]{2}{*}{ SEM } & \multirow[b]{2}{*}{$\begin{array}{c}\text { P- } \\
\text { value }\end{array}$} \\
\hline & $\begin{array}{l}\text { Recommended } \\
\text { Energy }\end{array}$ & $\begin{array}{c}\text { Low } \\
\text { Energy }\end{array}$ & & & $\begin{array}{c}\text { Canola } \\
\text { Oil }\end{array}$ & $\begin{array}{c}\text { Fish } \\
\text { Oil }\end{array}$ & $\begin{array}{c}\text { Palm } \\
\text { Oil }\end{array}$ & $\begin{array}{c}\text { Poultry } \\
\text { Oil }\end{array}$ & & \\
\hline \multicolumn{11}{|c|}{ Starter Phase (8-21 days) } \\
\hline Bird cost & 34.0 & 34.0 & - & - & 34.0 & 34.0 & 34.0 & 34.0 & - & - \\
\hline $\begin{array}{l}\text { Average feed } \\
\text { intake }(\mathrm{g})\end{array}$ & 1112.3 & 1135.2 & 20.5 & 0.438 & 1123.7 & 1117.7 & 1147.6 & 1105.8 & 29.0 & 0.777 \\
\hline Feed cost / kg & 55.9 & 52.7 & - & - & 54.1 & 56.0 & 54.0 & 53.1 & - & - \\
\hline Feed cost / bird & 62.2 & 59.8 & 1.12 & 0.140 & 60.8 & 62.6 & 61.9 & 58.7 & 1.58 & 0.342 \\
\hline \multicolumn{11}{|c|}{ Finisher Phase (22-35 days) } \\
\hline $\begin{array}{l}\text { Average feed } \\
\text { intake }(\mathrm{g})\end{array}$ & 2067.9 & 2094.8 & 28.9 & 0.516 & $1978.8^{b}$ & $2187.2^{\mathrm{a}}$ & $2131.3^{\mathrm{ab}}$ & $2028.3^{\mathrm{ab}}$ & 40.9 & 0.006 \\
\hline Feed cost / kg & 55.7 & 52.6 & - & - & 54.3 & 57.3 & 53.3 & 51.6 & - & - \\
\hline Feed cost / bird & $115.3^{\mathrm{a}}$ & $110.1^{b}$ & 1.53 & 0.026 & $107.5^{\mathrm{bc}}$ & $125.3^{\mathrm{a}}$ & $113.6^{\mathrm{b}}$ & $104.5^{\mathrm{c}}$ & 2.17 & 0.0001 \\
\hline \multicolumn{11}{|c|}{ Overall Period (8-35 days) } \\
\hline Feed cost / bird & $177.5^{\mathrm{a}}$ & $169.9^{\mathrm{b}}$ & 1.68 & 0.004 & $168.3^{\mathrm{bc}}$ & $187.9^{\mathrm{a}}$ & $175.5^{\mathrm{b}}$ & $163.2^{\mathrm{c}}$ & 2.38 & 0.0001 \\
\hline Miscellaneous $^{1}$ & 25.0 & 25.0 & - & - & 25.0 & 25.0 & 25.0 & 25.0 & - & - \\
\hline $\begin{array}{l}\text { Production cost } \\
/ \text { bird }^{2}\end{array}$ & $236.5^{\mathrm{a}}$ & $228.9^{\mathrm{b}}$ & 1.68 & 0.004 & $227.3^{\mathrm{bc}}$ & $246.9^{\mathrm{a}}$ & $234.5^{\mathrm{b}}$ & $222.2^{\mathrm{c}}$ & 2.38 & 0.0001 \\
\hline $\begin{array}{l}\text { Average body } \\
\text { weight }(\mathrm{g})\end{array}$ & $1964.4^{\mathrm{a}}$ & $1914.2^{\mathrm{b}}$ & 12.4 & 0.008 & $1958.0^{\mathrm{a}}$ & $\begin{array}{c}1935.7^{\mathrm{a}} \\
\mathrm{b}\end{array}$ & $1887.4^{\mathrm{b}}$ & $1976.2^{\mathrm{a}}$ & 17.5 & 0.009 \\
\hline $\begin{array}{l}\text { Production cost } \\
/ \mathrm{kg}\end{array}$ & 120.7 & 119.6 & 1.02 & 0.451 & $116.1^{\mathrm{b}}$ & $127.6^{\mathrm{a}}$ & $124.3^{\mathrm{a}}$ & $112.6^{\mathrm{b}}$ & 1.44 & 0.0001 \\
\hline
\end{tabular}

Table 11. Effect of energy levels and oil sources on economics efficiency (Simple effect).

\begin{tabular}{|c|c|c|c|c|c|c|c|c|c|c|}
\hline \multirow[b]{2}{*}{ Production Cost (Rs.) } & \multicolumn{4}{|c|}{ Recommended Energy } & \multicolumn{4}{|c|}{ Low Energy } & \multirow[b]{2}{*}{ SEM } & \multirow[b]{2}{*}{$\begin{array}{c}\text { P- } \\
\text { value }\end{array}$} \\
\hline & $\begin{array}{c}\text { Canola } \\
\text { Oil }\end{array}$ & $\begin{array}{c}\text { Fish } \\
\text { Oil }\end{array}$ & $\begin{array}{c}\text { Palm } \\
\text { Oil }\end{array}$ & $\begin{array}{c}\text { Poultry } \\
\text { Oil }\end{array}$ & $\begin{array}{c}\text { Canola } \\
\text { Oil }\end{array}$ & $\begin{array}{c}\text { Fish } \\
\text { Oil }\end{array}$ & $\begin{array}{c}\text { Palm } \\
\text { Oil }\end{array}$ & $\begin{array}{c}\text { Poultry } \\
\text { Oil }\end{array}$ & & \\
\hline \multicolumn{11}{|c|}{ Starter Phase (8-21 days) } \\
\hline Bird cost & 34 & 34 & 34 & 34 & 34 & 34 & 34 & 34 & - & - \\
\hline Average feed intake $(\mathrm{g})$ & 1117.9 & 1124.4 & 1121.0 & 1085.8 & 1129.5 & 1111.1 & 1174.3 & 1125.8 & 41.1 & 0.852 \\
\hline Feed cost / kg & 56.0 & 58.4 & 55.3 & 53.9 & 52.2 & 53.5 & 52.7 & 52.3 & - & - \\
\hline Feed cost / bird & 62.6 & 65.7 & 62.0 & 58.5 & 58.9 & 59.5 & 61.9 & 58.9 & 2.24 & 0.417 \\
\hline \multicolumn{11}{|c|}{ Finisher Phase (22-35 days) } \\
\hline Average feed intake (g) & $2096.0^{\mathrm{abc}}$ & $2185.7^{\mathrm{ab}}$ & $2030.5^{\mathrm{abc}}$ & $1959.5^{\mathrm{bc}}$ & $1861.6^{\mathrm{c}}$ & $2188.7^{\mathrm{ab}}$ & $2232.0^{\mathrm{a}}$ & $2097.1^{\mathrm{abc}}$ & 57.8 & 0.005 \\
\hline Feed cost / kg & 55.8 & 59.8 & 54.6 & 52.3 & 52.7 & 54.7 & 52.0 & 50.8 & - & - \\
\hline Feed cost / bird & $117.1^{\mathrm{ab}}$ & $130.8^{\mathrm{a}}$ & $111.0^{\mathrm{bcd}}$ & $102.5^{\mathrm{cd}}$ & $98.0^{\mathrm{d}}$ & $119.8^{\mathrm{ab}}$ & $116.1^{\mathrm{bc}}$ & $106.6^{\mathrm{bcd}}$ & 3.06 & 0.001 \\
\hline \multicolumn{11}{|c|}{ Overall Period (8-35 days) } \\
\hline Feed cost / bird & $179.7^{\mathrm{b}}$ & $196.5^{\mathrm{a}}$ & $172.9^{\mathrm{bc}}$ & $161.0^{\mathrm{cd}}$ & $157.0^{\mathrm{d}}$ & $179.3^{\mathrm{b}}$ & $178.0^{\mathrm{b}}$ & $165.4^{\mathrm{bcd}}$ & 3.37 & 0.0001 \\
\hline Miscellaneous ${ }^{1}$ & 25.0 & 25.0 & 25.0 & 25.0 & 25.0 & 25.0 & 25.0 & 25.0 & - & - \\
\hline Production cost / bird ${ }^{2}$ & $238.7^{\mathrm{b}}$ & $255.5^{\mathrm{a}}$ & $231.9^{\mathrm{bc}}$ & $220.0^{\mathrm{cd}}$ & $216.0^{d}$ & $238.3^{\mathrm{b}}$ & $237.0^{\mathrm{b}}$ & $224.4^{\mathrm{bcd}}$ & 3.37 & 0.0001 \\
\hline Average body weight (g) & $2009.6^{\mathrm{ab}}$ & $1939.1^{\mathrm{abc}}$ & $1865.4^{\mathrm{c}}$ & $2043.3^{\mathrm{a}}$ & $1906.3^{\mathrm{bc}}$ & $1932.2^{\mathrm{abc}}$ & $1909.3^{\mathrm{bc}}$ & $1909.2^{\mathrm{bc}}$ & 24.7 & 0.005 \\
\hline Production cost / kg & $118.8^{\mathrm{bc}}$ & $131.8^{\mathrm{a}}$ & $124.5^{\mathrm{ab}}$ & $107.7^{\mathrm{d}}$ & $113.4^{\mathrm{cd}}$ & $123.3^{\mathrm{ab}}$ & $124.1^{\mathrm{ab}}$ & $117.6^{\mathrm{bc}}$ & 2.04 & 0.001 \\
\hline
\end{tabular}

${ }^{1}$ Miscellaneous cost include vaccination cost, farm preparation and brooding expenditures

${ }^{2}$ Production cost per bird = Bird cost + Feed cost per bird + Miscellaneous; a-d values of superscript different in row differ significantly 


\section{DISCUSSION}

Weight gain, PER, EPEF, FE and FCR were improved $(\mathrm{P} \leq 0.05)$ in birds received diet having poultry oil and canola oil as compared to those fed diets having fish and palm oil due to the highest level of unsaturated fatty acids in canola oil which are converted by birds to omega-3 fatty acids. Further, lower level of FFA and POV in poultry oil increased its digestion and absorption unlike fish oil which had higher value of FFA and POV leads to poor performance (table 3). In accordance with present study, it was reported that the addition of poultry fat and canola oil at 3\% in broiler diet had higher weight gain and better FCR than those receiving $6 \%$ canola oil and poultry fat alone or in combination (Shahryar et al., 2011). This might be due to the higher amount of metabolizable energy in canola oil and the highest capability of digestion and absorption of unsaturated fatty acids that exists in canola oil (as a high content) is the main factor that birds can keep their energy received with reduction of feed consumption. Likewise, use of vegetable oil like sunflower oil in broiler diet had greater WG and improved FCR than those fed palm oil (Khatun et al., 2018). Broiler birds fed diet containing soybean oil had higher WG and better FCR than those fed poultry fat and tallow oil (Zhang et al., 2011). Contrarily, no effect of chicken tallow and soybean oil in broiler were observed on FI, WG and FCR (Polycarpo et al., 2014). Birds fed diet containing beef tallow and canola oil had similar WG, FI and FCR (Meng et al., 2004).

Higher $(\mathrm{P} \leq 0.05)$ dressing \% was observed in birds fed recommended energy diet than those fed low energy diet. Probably birds fed diet having low energy diet had higher proportion of visceral organs resulting in lower dressing percentage. Breast yield, thigh weight, heart weight and gizzard weight were similar in birds fed different energy levels and oil sources. Addition of poultry fat and canola oil in broiler diet had lower gizzard weight and higher abdominal fat, while, breast yield, thigh yield and liver weight were no affected by oil sources (Shahryar et al., 2011). Different fat sources (chicken tallow and soybean oil) in broiler diet had similar effect on carcass yield; drumstick and chest yield (Polycarpo et al., 2014). Soybean oil and poultry fat in broiler diet had similar effect on carcass, breast and thigh yield (Neto et al., 2011). Use of palm oil, sunflower oil and their combination in broiler diet had similar effect on breast muscle $\mathrm{pH}$, cooking loss and breast meat color (Khatun et al., 2018). Liver weight was higher in birds fed fish oil-based diet. This might be due to that fish oil contains higher content to free fatty acid which causes oxidation in liver which cause increase in size.

Different oil sources (canola, palm, refined poultry and fish oil) had no effect on DM, EE and CP digestibility at 21 day. Similar outcomes were noted by Abdulla et al. (2016) who revealed that different fat sources (soybean, palm and linseed) in broiler diet had similar organic matter, ether extract, crude protein and ash digestibility. Further, different oil sources (beef tallow and canola oil) in broiler diet had similar effect on fat, starch and nitrogen digestibility (Meng et al., 2004).

Birds fed diet having poultry and canola oil had higher DM, CP and EE digestibility at $35^{\text {th }}$ day than those fed diet having palm and fish oil. This is due to that canola and poultry oil had lower adulteration and higher caloric value unlike fish oil which had higher value of FFA and POV value and lower digestibility. Results are in line with the outcome of Polycarpo et al. (2014) who observed that vegetable oil like soybean oil in broiler diet resulted in greater digestibility coefficient of nitrogen in broiler birds than those fed chicken tallow. Apparent digestibility coefficient of fat in birds fed soybean oil was higher than those received tallow oil (Tancharoenrat et al., 2014). Higher net profit in birds fed diet having poultry oil is due to higher body weight and low price or poultry oil as compared to canola, palm and fish oil.

Conclusion: It was concluded from the results that birds fed diet having canola and poultry oil had improved growth performance, dressing percentage and meat quality than those fed palm oil and fish oil. However, in term of economics, addition of refined poultry oil in recommended energy diet is beneficial.

Acknowledgments: The authors acknowledge the fellowship provided by the Higher Education Commission of Pakistan (HEC) to Muhammad Shoaib under the framework of HEC Indigenous PhD Fellowship Program.

\section{REFERENCES}

Abdulla, N., T. Loh, H. Akit, A. Sazili and H. Foo (2016). Effects of dietary oil sources and calcium: phosphorus levels on growth performance, gut morphology and apparent digestibility of broiler chickens. S. Afr. J. Anim. Sci. 46: 42-53.

Ahmed H.O., Z. Hassan, and M.N.A. Manap (2015). Effect of slaughtering methods on meat quality indicators, chemical changes and microbiological quality of broiler chicken meat during refrigerated storage. J. Agric. Vet. Sci. 8: 12-17.

Allahyari-Bake, S., and R. Jahanian (2017). Effects of dietary fat source and supplemental lysophosphatidylcholine on performance, immune responses, and ileal nutrient digestibility in broilers fed corn/soybean meal or corn/wheat/ soybean meal based diets. Poult. Sci. 96:1149-1158.

AOAC (2000). Official Methods of Analysis. (17 $7^{\text {th }}$ Edition). Association of Official Analytical Chemists. Maryland, U.S.A.

Baião, N., and L. Lara (2005). Oil and fat in broiler nutrition. Braz. J. Poult. Sci. 7:129-141.

Butolo, J (2002). Quality of ingredients in animal feed. 
Campinas: Brazilian College of Animal Nutrition. 2: $430-438$.

Classen, H.L (2017). Diet energy and feed intake in chickens. Anim. Feed Sci. Technol. 233:13-21.

Dourado, L.R.B., J.C. Siqueira, N.K. Sakomura, S.R.F. Pinheiro, S.M. Marcato, J.B.K. Fernandes, and J.H.V. Silva (2010). Poultry feed metabolizable energy determination using total or partial excreta collection methods. Braz. J. Poult. Sci. 12:129132.

Fan, H.P., M. Xie, W.W. Wang, S.S. Hou, and W. Huang (2008). Effects of dietary energy on growth performance and carcass quality of white growing Pekin ducks from two to six weeks of age. Poult. Sci. 87:1162-1164.

Firman, J.D., A. Kamyab, and H. Leigh (2008). Comparison of fat sources in rations of broilers from hatch to market. Int. J. Poult. Sci. 7:1152-1155.

Jeacocke, R.E (1977). Continuous measurement of the $\mathrm{pH}$ of beef muscle in intact beef carcasses. J. Food Technol. 12:375-386.

Kamran, Z., M. Sarwar, M. Nisa, M.A. Nadeem, S. Mahmood, M.E. Babar, and S. Ahmed (2008). Effect of low-protein diets having constant energyto-protein ratio on performance and carcass characteristics of broiler chickens from one to thirty-five days of age. Poult. Sci. 87:468-474.

Khatun, J., T. C. Loh, H. Akit, H. L. Foo, and R. Mohamad (2018). Influence of different sources of oil on performance, meat quality, gut morphology, ileal digestibility and serum lipid profile in broilers. J. Appl. Anim. Res. 46: 479-485.

Kiani, A., S. D. Sharifi, and G. Shokoufeh (2017). Influence of canola oil and lysine supplementation diets on growth performance and fatty acid composition of meat in broiler chicks. Int. J. Anim. Vet. Sci. 11: 134-140.

Liu, W., R. Pedram, F. Javandel, S. Nasabian, and A. Seidavi (2017). The effects of different levels of dietary fish oil, soybean oil, and sunflower oil on performance and immunity related parameters of broiler chicken. J. Anim. Plant Sci. 27:384-388.

Long, S., Y. Xu, C. Wang, C. Li, D. Liu, and X. Piao (2018). Effects of dietary supplementation with a combination of plant oils on performance, meat quality and fatty acid deposition of broilers. AsianAust. J. Anim. Sci. 31:1773-1780.

Marcu, A., I. Vacaru, D. Gabi, P.C. Liliana, A. Marcu, N. Marioara, P. Ioan, D. Dorel, K. Bartolomeu, and M. Cosmin (2013). The influence of genetics on economic efficiency of broiler chickens growth. Anim. Sci. Biotechnol. 46:339 346.

Meng, X., B. A. Slominski, and W. Guenter (2004). The effect of fat type, carbohydrase, and lipase addition on growth performance and nutrient utilization of young broilers fed wheat-based diets. Poult. Sci. 83: 1718-1727.

Neto, G., A. Pezzato, J. Sartori, C. Mori, V. Cruz, V. Fascina, D. Pinheiro, L. Madeira, and J. Gonçalvez (2011). Emulsifier in broiler diets containing different fat sources. Braz. J. Poult. Sci. 13: 119125.

Pearson, A.M., and T.R. Dutson (1995). Quality attributes and their measurement in meat, poultry and fish products. Springer: Berlin, Germany.

Polycarpo, G.V., V.C. Cruz, N.C. Alexandre, V.B. Fascina, I.M.G.P. Souza, J.C.M. Cravo, R. Albuquerque, J.R. Sartori, and A.C. Pezzato (2014). Effect of lipid sources and inclusion levels in diets for broiler chickens. Arq. Bras. Med. Vet. e Zootec. 66: 519-528.

Shahryar, H.A., R. Salamatdoust, A. Lak, and A. Lotfi (2011). Effect of dietary supplemented canola oil and poultry fat on the performance and carcass characterizes of broiler chickens. J. Biol. Sci. 3: 388-392.

Skrivan, M., M. Marounek, M. Englmaierová, L. Cermák, J. Vlčková, and E. Skrivanová (2018). Effect of dietary fat type on intestinal digestibility of fatty acids, fatty acid profiles of breast meat and abdominal fat, and mRNA expression of lipidrelated genes in broiler chickens. PLoS ONE 13:e0196035.

Smits, C.H.M., P.J. Moughan, and A.C. Beynen (2000). The inhibitory effect of a highly viscous carboxymethylcellulose on dietary fat digestibility in the growing chicken is dependent on the type of fat. J. Anim. Physiol. Anim. Nutr. 83: 231-238.

Steel, R.G.D., J.H. Torrie, and D.A. Dickie (1997). Principles and Procedures of Statistics. A Biometric Approach, 3rd edition, McGraw-Hill, Book Publishing Company, Toronto, Canada.

Tancharoenrat, P., V. Ravindran, F. Zaefarian, and G. Ravindran (2014). Digestion of fat and fatty acids along the gastrointestinal tract of broiler chickens. Poult. Sci. 93: 371-379.

Zhang, B., L. Haitao, D. Zhao, Y. Guo, and A. Barri (2011). Effect of fat type and lysophosphatidylcholine addition to broiler diets on performance, apparent digestibility of fatty acids, and apparent metabolizable energy content. Anim. Feed Sci. Technol. 163: 177-184.

Zhao, P.Y., and I.H. Kim (2017). Effect of diets with different energy and lipase levels on performance, digestibility and carcass trait in broilers. Poult. Sci. 96:1341-1347. 